Article

\title{
Uniqueness for Inverse Source Problems of Determining a Space-Dependent Source in Time-Fractional Equations with Non-Smooth Solutions
}

\author{
Karel Van Bockstal
}

check for

updates

Citation: Van Bockstal, $\mathrm{K}$

Uniqueness for Inverse Source

Problems of Determining a

Space-Dependent Source in

Time-Fractional Equations with

Non-Smooth Solutions. Fractal Fract.

2021, 5, 169. https://doi.org/10.3390/

fractalfract5040169

Academic Editor: Ricardo Almeida

Received: 19 August 2021

Accepted: 10 October 2021

Published: 16 October 2021

Publisher's Note: MDPI stays neutral with regard to jurisdictional claims in published maps and institutional affiliations.

Copyright: (C) 2021 by the author. Licensee MDPI, Basel, Switzerland. This article is an open access article distributed under the terms and conditions of the Creative Commons Attribution (CC BY) license (https:// creativecommons.org/licenses/by/ $4.0 /)$.
Research Group NaM², Department of Electronics and Information Systems, Ghent University, Krijgslaan 281, 9000 Ghent, Belgium; karel.vanbockstal@ugent.be; Tel.: +32-9-264-49-10

\begin{abstract}
In this contribution, we investigate an inverse source problem for a fractional diffusion and wave equation with the Caputo fractional derivative of the space-dependent variable order. More specifically, we discuss the uniqueness of a solution when reconstructing a space-dependent source from a time-averaged measurement, or a final in time measurement. Weakly singular solutions are included in the class of admissible solutions. The obtained results are also valid if the order of the fractional derivative is constant.
\end{abstract}

Keywords: time-fractional diffusion equation; non-autonomous; inverse source problem; uniqueness

\section{Introduction}

\subsection{Mathematical Formulation of the Problem}

In this paper, we consider a bounded domain $\Omega \subset \mathbb{R}^{d}, d \in \mathbb{N}$ with a Lipschitz continuous boundary $\partial \Omega$. We denote the final time by $T$ and define $Q_{T}:=\Omega \times(0, T]$ and $\Sigma_{T}:=\partial \Omega \times(0, T]$. Further, we consider a general second-order linear differential operator $L(t)$ defined by:

$$
L(t) u(\mathbf{x}, t)=-\nabla \cdot(\boldsymbol{A}(\mathbf{x}, t) \nabla u(\mathbf{x}, t))+c(\mathbf{x}, t) u(\mathbf{x}, t),
$$

where

$$
\boldsymbol{A}(\mathbf{x}, t)=\left(a_{i, j}(\mathbf{x}, t)\right)_{i, j=1, \ldots, d^{\prime}} \quad \mathbf{x} \in \Omega, \quad \boldsymbol{A}=\boldsymbol{A}^{\top} .
$$

We involve the Caputo fractional derivative of the space-dependent variable order in the form (see, e.g., ([1], Equation (1.6)):

$$
\begin{array}{r}
{ }^{n} D_{t}^{\beta(\mathbf{x})} u(\mathbf{x}, t):=\frac{1}{\Gamma(n-\beta(\mathbf{x}))} \partial_{t} \int_{0}^{t}(t-s)^{n-\beta(\mathbf{x})-1}\left(\partial_{s}^{n-1} u(s, \mathbf{x})-\left.\partial_{s}^{n-1} u(s, \mathbf{x})\right|_{s=0}\right) \mathrm{d} s \\
n-1<\beta(\mathbf{x}) \leqslant \beta_{n}<n, \quad n=1,2,
\end{array}
$$

where $\beta \in \mathrm{C}(\bar{\Omega})$. The inverse source problem (ISP) here is to search the couple $\{f(\mathbf{x}), u\}$ for given $h(t), F, g, \tilde{u}_{0}$ and $\tilde{v}_{0}$ (if $n=2$ ), such that:

$$
\left\{\begin{aligned}
{ }^{n} D_{t}^{\beta(\mathbf{x})} u(\mathbf{x}, t)+L u(\mathbf{x}, t) & =f(\mathbf{x}) h(t)+F(\mathbf{x}, t) & (\mathbf{x}, t) \in Q_{T}, \\
u(\mathbf{x}, t) & =g(\mathbf{x}, t) & (\mathbf{x}, t) \in \Sigma_{T}, \\
u(\mathbf{x}, 0) & =\tilde{u}_{0}(\mathbf{x}) & \mathbf{x} \in \Omega, \\
\partial_{t} u(\mathbf{x}, 0) & =\tilde{v}_{0}(\mathbf{x}) & \mathbf{x} \in \Omega, \text { if } n=2,
\end{aligned}\right.
$$


along with the time-averaged measurement:

$$
\int_{0}^{T} u(\mathbf{x}, t) \mathrm{d} t=U_{T}(\mathbf{x}), \quad \mathbf{x} \in \Omega,
$$

or with the given final in time measurement:

$$
u(\mathbf{x}, T)=\psi_{T}(\mathbf{x}), \quad \mathbf{x} \in \Omega .
$$

In the case $n=1$ (i.e., $\left.0<\beta(\mathbf{x}) \leqslant \beta_{1}<1\right)$, the governing equation is called a fractional diffusion equation of space-dependent order, whilst the equation is called a fractional wave equation of space-dependent order in the case $n=2$ (i.e., $1<\beta(\mathbf{x}) \leqslant \beta_{2}<2$ ). For $n=1,2$, we define:

$$
g_{n}: Q_{T} \rightarrow \mathbb{R}:(\mathbf{x}, t) \mapsto g_{n}(\mathbf{x}, t)=\frac{t^{n-\beta(\mathbf{x})-1}}{\Gamma(n-\beta(\mathbf{x}))} .
$$

With the use of these kernels, as for the constant order case, we can rewrite ${ }^{n} D_{t}^{\beta(\mathbf{x})}$ as:

$$
{ }^{n} D_{t}^{\beta(\mathbf{x})} u(\mathbf{x}, t)= \begin{cases}\partial_{t}\left(g_{1} *\left(u-\tilde{u}_{0}\right)\right)(\mathbf{x}, t) & \text { if } n=1, \\ \partial_{t}\left(g_{2} *\left(\partial_{t} u-\tilde{v}_{0}\right)\right)(\mathbf{x}, t) & \text { if } n=2,\end{cases}
$$

where the symbol ' $*$ ' stands for the classical convolution product defined by $(f * g)(t)=$ $\int_{0}^{t} f(t-s) g(s) \mathrm{d} s$. This convolution structure is crucial when establishing the result in [2] (Lemma 2.1), which is used throughout this paper.

\subsection{Literature Overview}

The determination of a space-dependent source from Measurements (3) and (4) in evolutionary (parabolic or hyperbolic) partial differential equations (PDEs) has been the subject of many studies; see, e.g., [3-10] and the references therein. Many recent studies have been devoted to the recognition of the space-dependent part of the source for fractional partial differential equations (FPDEs); we refer to [11-16]. These investigations deal with ISPs for fractional equations of constant order, whilst, in this contribution, fractional equations of space-dependent order are considered (from which the constant order is a special case). Now, we will discuss these articles in more detail.

In $[11,12]$, the uniqueness of a solution to ISP (2), (4) with $L(t)=L$ (i.e., $L$ is autonomous) is established via the Mittag-Leffler analysis. In particular, [12] (Theorem 3.1) shows that if $h \in \mathrm{C}([0, T])$ satisfies $h(t) \geqslant h_{0}>0$, then the solution to this ISP is unique. A similar problem with time-dependent coefficients in operator $L$ (i.e., $L$ is non-autonomous) is considered in [13] by using a variational approach. Uniqueness was established under a monotonicity assumption on $h$ (next to some monotonicity assumptions on the coefficients in $L(t))$, i.e., $\left\{h>0, h^{\prime} \geqslant 0\right\}$ or $\left\{h<0, h^{\prime} \leqslant 0\right\}$. However, this study does not deal with the typical behaviour of solutions to time-fractional diffusion equations [17]. In general, it has been shown that the solution to such a problem has a weak singularity near the initial time $t=0$. This means that $\partial_{t} u$ blows up as $t \searrow 0$ for $n=1[17,18]$, and $\partial_{t t} u$ blows up as $t \searrow 0$ for $n=2$ [19]. In particular, in [13] (Theorem 3.1), it is required that $\partial_{t} u \in \mathrm{L}^{2}(0, T)$, which is not generally the case for solutions to time-fractional diffusion equations. The same issue is encountered in [16] (Theorem 3.1), where the author considers a non-autonomous operator $L(t)$ in the form $L(t) u(\mathbf{x}, t)=p(t)[-\nabla \cdot(\boldsymbol{A}(\mathbf{x}) \nabla u(\mathbf{x}, t))+c(\mathbf{x}) u(\mathbf{x}, t)]$ with $p>0$. By the separation of variable techniques combined with a maximum principle, the author establishes the uniqueness of a solution if $h \in \mathrm{C}([0, T])$ satisfies $0 \not \equiv h \geqslant 0$, and states that $\partial_{t} u \in \mathrm{L}^{2}(0, T)$.

Up to now, little attention has been paid to the case $n=2$ (fractional wave equation). In [14], the authors identified $f(\mathbf{x})$ uniquely on the basis of $\left.u\right|_{\Gamma^{\prime} \times(0, T)}$ with the help of the Duhamel principle and Tikhonov regularization method, whilst the authors in [15] studied 
the recovery of $f(\mathbf{x})$ from (4) using the Landweber method. However, no uniqueness of the solution to the ISP is obtained.

\subsection{Objectives}

The importance and originality of this study are that it explores which type of measurement is suited to recover, in a unique way, a space-dependent source $f(\mathbf{x})$, taking into account the typical behaviour of the solution to FPDEs. In comparison with [11], the coefficients in the governing operator $L$ are considered to be time-dependent and the Caputo fractional derivative is of a space-dependent variable order. Moreover, for the first time in the literature, Measurement (3) is considered for FPDEs. The outline of the paper is as follows:

In Section 2, we discuss the case $n=1$ (i.e., fractional diffusion equation of spacedependent variable order). First, we consider Measurement (3), and make a distinction between $L$ autonomous and non-autonomous. Moreover, we investigate whether $f(\mathbf{x})$ can be uniquely determined from the Measurement (4) if the solution $u$ of problem (2) satisfies the bounds:

$$
\left|\frac{\partial^{l} u}{\partial t^{l}}(\mathbf{x}, t)\right|+\left|\nabla \frac{\partial^{l} u}{\partial t^{l}}(\mathbf{x}, t)\right| \leqslant C\left(1+t^{\beta_{1}-l}\right) \quad \text { for } l=0,1 \quad \text { for a.a. }(\mathbf{x}, t) \in Q_{T} .
$$

Note that $\beta_{1}=\beta$ if $\beta(\mathbf{x})=\beta$. In the latter case, this realistic assumption in Solution (5) can be found in, e.g., $[18,20]$.

In Section 3, the aim is to investigate whether both measurements are suited for the reconstruction of $f(\mathbf{x})$ if $n=2$ (i.e., fractional wave equation of space-dependent variable order). The results will depend on the properties of the operator $L$. The solution $u$ can satisfy the bounds:

$$
\left|\frac{\partial^{l} u}{\partial t^{l}}(\mathbf{x}, t)\right|+\left|\nabla \frac{\partial^{l} u}{\partial t^{l}}(\mathbf{x}, t)\right| \leqslant C\left(1+t^{\beta_{2}-l}\right) \quad \text { for } l=0,1,2 \quad \text { for a.a. }(\mathbf{x}, t) \in Q_{T},
$$

see Section 4.1 in [19] for $\beta(\mathbf{x})=\beta$ (then, $\beta_{2}=\beta$ ).

Finally, Section 4 presents the findings of the research, and several directions for future work are mentioned.

\section{Inverse Source Problem for $\boldsymbol{n}=\mathbf{1}$}

In this section, we studied ISPs (2), (3) and (2), (4) using a variational approach. We used the following lemmas (see [2] (Lemma 2.1), [2] (Corollary 3.1), and [2] (Theorem 6.1)) to study the uniqueness of the solution to the ISPs.

Lemma 1 (Strongly positive definiteness). For all $v \in \mathrm{L}_{\mathrm{loc}}^{2}\left((0, \infty), \mathrm{L}^{2}(\Omega)\right)$, for $n=1,2$, the kernel $g_{n}$ satisfies:

$$
\int_{0}^{t}\left(\left(g_{n} * v\right)(s), v(s)\right) \mathrm{d} s \geqslant \int_{\Omega} \gamma_{n}(\mathbf{x}) \int_{0}^{t}(e * v)^{2}(\mathbf{x}, s) \mathrm{d} s \mathrm{~d} \mathbf{x},
$$

with

$$
e(t)=\mathrm{e}^{-t} \quad \text { and } \quad \gamma_{n}(\mathbf{x})=\cos \left([n-\beta(\mathbf{x})] \frac{\pi}{2}\right)
$$

Lemma 2 (Energy estimate). For any $v:[0, T] \rightarrow \mathrm{L}^{2}(\Omega)$ satisfying:

$$
v \in \mathrm{L}^{2}\left((0, T), \mathrm{L}^{2}(\Omega)\right) \quad \text { with } \quad g_{n} * v \in \mathrm{H}^{1}\left((0, T), \mathrm{L}^{2}(\Omega)\right),
$$


it holds for all $\eta \in[0, T]$ that there exists a strict positive constant $\tilde{c}_{n}$ such that:

$$
\int_{0}^{\eta}\left(\partial_{t}\left(g_{n} * v\right)(t), v(t)\right) \mathrm{d} t \geqslant \tilde{c}_{n} \int_{0}^{\eta}\|v(t)\|^{2} \mathrm{~d} t, \quad \tilde{c}_{n}:= \begin{cases}\frac{\min \left\{1, T^{\left.-\beta_{1}\right\}}\right.}{2 \Gamma\left(1-\beta_{1}\right)} & \text { if } n=1, \\ \frac{\min \left\{1, T^{\left.1-\beta_{2}\right\}}\right.}{2 \Gamma\left(2-\beta_{2}\right)} & \text { if } n=2 .\end{cases}
$$

Lemma 3 (Existence of a solution to the forward problem for $n=1$ ). Let the following assumptions be fulfilled:

- AS-1: $\boldsymbol{A}=\left(a_{i j}(\mathbf{x}, t)\right) \in\left(\mathrm{L}^{\infty}\left(Q_{T}\right)\right)^{d \times d}$ satisfying $\boldsymbol{A}^{T}=\boldsymbol{A}$ and $\boldsymbol{A}$ is uniformly elliptic;

- $\quad A S-2: c \in \mathrm{L}^{\infty}\left(Q_{T}\right)$ satisfying $c(\mathbf{x}, t) \geqslant 0$ for a.a. $(\mathbf{x}, t) \in Q_{T}$;

- AS-3: $\partial_{t} A \in\left(\mathrm{L}^{\infty}\left(Q_{T}\right)\right)^{d \times d}$ and $\partial_{t} c \in \mathrm{L}^{\infty}\left(Q_{T}\right)$;

- $\quad A S-4: f \in \mathrm{H}^{1}\left((0, T), \mathrm{L}^{2}(\Omega)\right)$ or $\left\|\frac{\partial^{\ell}}{\partial t^{\ell}} f(t)\right\| \leqslant C\left(1+t^{\alpha-\ell}\right)$ for $\ell=0,1$ and $\alpha \in(0,1)$;

- $\quad A S-5: \tilde{u}_{0} \in \mathrm{H}_{0}^{1}(\Omega)$.

Then, there exists a unique weak solution $u$ to Problem (2), with $u \in \mathrm{L}^{\infty}\left((0, T), \mathrm{H}_{0}^{1}(\Omega)\right)$ and $\partial_{t}\left(g_{1} *\left(u-\tilde{u}_{0}\right)\right) \in \mathrm{L}^{\infty}\left((0, T), \mathrm{H}_{0}^{1}(\Omega)^{*}\right)$.

From this lemma, we were not able to conclude that $u(\cdot, T) \in \mathrm{L}^{2}(\Omega)$. We first considered the time-average condition $\int_{0}^{T} u(\cdot, t) \mathrm{d} t=U_{T}(\cdot)$ for $n=1$, which was well-defined as an element of $\mathrm{L}^{2}(\Omega)$.

Theorem 1 (Uniqueness if $n=1$ with $L(t)=L$ using measurement (3)). Let the conditions of Lemma 3 be satisfied. Assume that $L(t)=L$ and $U_{T} \in \mathrm{L}^{2}(\Omega)$. Moreover, assume that $h \in \mathrm{L}^{1}(0, T)$ satisfies:

$$
h(t)>0, \quad \forall t \in(0, T), \quad \text { or either } \quad h(t)<0, \quad \forall t \in(0, T) .
$$

Then, there exists, at most, one couple $\langle u, f\rangle \in \mathrm{L}^{\infty}\left((0, T), \mathrm{H}_{0}^{1}(\Omega)\right) \times \mathrm{L}^{2}(\Omega)$ with $\partial_{t}\left(g_{1} *\left(u-\tilde{u}_{0}\right)\right) \in$ $\mathrm{L}^{\infty}\left((0, T), \mathrm{H}_{0}^{1}(\Omega)^{*}\right)$, such that Problem (2), together with Condition (3), is satisfied.

Proof. We supposed that there were two solutions $\left\{u_{1}, f_{1}\right\}$ and $\left\{u_{2}, f_{2}\right\}$ satisfying (2) and (3). Then, the differences $u=u_{1}-u_{2}$ and $f=f_{1}-f_{2}$ satisfied the weak form:

$$
\left\langle\partial_{t}\left(g_{1} * u\right)(t), \varphi\right\rangle+(A \nabla u(t), \nabla \varphi)+(c u(t), \varphi)=h(t)(f, \varphi),
$$

for all $\varphi \in \mathrm{H}_{0}^{1}(\Omega)$ with $u(\cdot, 0)=0$ and $\int_{0}^{T} u(\cdot, s) \mathrm{d} s=0$ in $\Omega$. Next, we integrated (7) in time over $t \in(0, \eta) \subset(0, T)$. Using [21] (Lemma 7.3), $\left(g_{1} * u\right)(\cdot, 0)=0$ almost everywhere (a.e.) in $\Omega$ and $\Phi_{h}(t):=\int_{0}^{t} h(s) \mathrm{d}$, we obtained:

$$
\left(\left(g_{1} * u\right)(\eta), \varphi\right)+\left(\int_{0}^{\eta} A \nabla u(t) \mathrm{d} t, \nabla \varphi\right)+\left(\int_{0}^{\eta} c u(t) \mathrm{d} t, \varphi\right)=\Phi_{h}(\eta)(f, \varphi) .
$$

First, we considered the case $h(t)>0$ for all $t \in(0, T)$, i.e., $\Phi_{h}(\eta)>0$ for all $\eta \in(0, T]$. We multiplied (8) by the reciprocal function $H(\eta):=\frac{1}{\Phi_{h}(\eta)}$, chose $\phi=u(\eta)$ as the test function, and integrated in time over $\eta \in(0, T)$ to obtain:

$$
\begin{aligned}
& \int_{0}^{T} H(\eta)\left(\left(g_{1} * u\right)(\eta), u(\eta)\right) \mathrm{d} \eta+\int_{0}^{T} H(\eta)\left(A \int_{0}^{\eta} \nabla u(t) \mathrm{d} t, \nabla u(\eta)\right) \mathrm{d} \eta \\
& \quad+\int_{0}^{T} H(\eta)\left(c \int_{0}^{\eta} u(t) \mathrm{d} t, u(\eta)\right) \mathrm{d} \eta=\int_{0}^{T}(f, u(\eta)) \mathrm{d} \eta=\left(f, \int_{0}^{T} u(\eta) \mathrm{d} \eta\right)=0 .
\end{aligned}
$$

Using integration by parts, we obtained for the first term on the left-hand side (LHS) of (9) that: 


$$
\begin{aligned}
& \int_{0}^{T} H(\eta)\left(\left(g_{1} * u\right)(\eta), u(\eta)\right) \mathrm{d} \eta \\
& \quad=H(T) \int_{0}^{T}\left(\left(g_{1} * u\right)(\eta), u(\eta)\right) \mathrm{d} \eta-\int_{0}^{T} H^{\prime}(\eta)\left(\int_{0}^{\eta}\left(\left(g_{1} * u\right)(t), u(t)\right) \mathrm{d} t\right) \mathrm{d} \eta .
\end{aligned}
$$

It was clear that $H^{\prime}(\eta)=-\frac{h(\eta)}{\Phi_{h}(\eta)^{2}} \leqslant 0$ for all $\eta \in[0, T]$. Therefore, with Lemma 1 ( $g_{1}$ is strongly positive definite), we obtained:

$$
\int_{0}^{T} H(\eta)\left(\left(g_{1} * u\right)(\eta), u(\eta)\right) \mathrm{d} \eta \geqslant H(T) \int_{\Omega} \gamma_{1}(\mathbf{x}) \int_{0}^{T}(e * u)^{2}(\mathbf{x}, s) \mathrm{d} s \mathrm{~d} \mathbf{x} .
$$

The second term on the LHS of (9) could be handled by employing $A=A^{\frac{1}{2}} A^{\frac{1}{2}}$ (note that a unique symmetric positive definite square root $A^{\frac{1}{2}}$ exists, since the symmetric matrix $A$ is positive, definite in space and time-cf. [22] (Theorem 1) —and integration by parts as follows:

$$
\begin{aligned}
\int_{0}^{T} H(\eta)\left(A \int_{0}^{\eta} \nabla u(t) \mathrm{d} t, \nabla u(\eta)\right) \mathrm{d} \eta & =\frac{1}{2} \int_{0}^{T} H(\eta)\left(\partial_{\eta}\left\|A^{\frac{1}{2}} \int_{0}^{\eta} \nabla u(t) \mathrm{d} t\right\|^{2}\right) \mathrm{d} \eta \\
& =-\frac{1}{2} \int_{0}^{T} H^{\prime}(\eta)\left\|A^{\frac{1}{2}} \int_{0}^{\eta} \nabla u(t) \mathrm{d} t\right\|^{2} \mathrm{~d} \eta \geqslant 0 .
\end{aligned}
$$

Analogously, we had:

$$
\int_{0}^{T} H(\eta)\left(c \int_{0}^{\eta} u(t) \mathrm{d} t, u(\eta)\right) \mathrm{d} \eta \geqslant 0
$$

Therefore, from (9), we obtained:

$$
H(T) \int_{\Omega} \gamma_{1}(\mathbf{x}) \int_{0}^{T}(e * u)^{2}(\mathbf{x}, s) \mathrm{d} s \mathrm{~d} \mathbf{x} \leqslant 0 .
$$

Hence, we obtained that $e * u=0$ a.e. in $Q_{T}$. This was a Volterra equation of the first kind. From [23] (Theorem 3.5 on p. 44), it followed that $u=0$ a.e. in $Q_{T}$. Hence, from (7), it followed that $(f, \varphi)=0$ for all $\varphi \in \mathrm{H}_{0}^{1}(\Omega)$. An application of [24] (Proposition 18.2) implied that $f=0$ in $\mathrm{L}^{2}(\Omega)$. The proof was similar if $h(t)<0$ for all $t \in(0, T)$, then (10) became:

$$
H(T) \int_{\Omega} \gamma_{1}(\mathbf{x}) \int_{0}^{T}(e * u)^{2}(\mathbf{x}, s) \mathrm{d} s \mathrm{~d} \mathbf{x} \geqslant 0,
$$

and the remainder of the proof proceeded as before.

If we assumed that solution $u$ satisfied additionally $\partial_{t}\left(g_{1} *\left(u-\tilde{u}_{0}\right)\right) \in \mathrm{L}^{\infty}\left((0, T), \mathrm{L}^{2}(\Omega)\right)$, then we could show the uniqueness of a solution to ISP (2) and (3), with time-dependent coefficients in operator $L$.

Theorem 2 (Uniqueness if $n=1$ using measurement (3)). Assume $U_{T} \in \mathrm{L}^{2}(\Omega)$. Moreover, assume that $h \in \mathrm{C}^{1}((0, T])$ satisfies:

$$
h(t)>0, \quad h^{\prime}(t) \geqslant 0, \quad \forall t \in(0, T),
$$

or, either,

$$
h(t)<0, \quad h^{\prime}(t) \leqslant 0, \quad \forall t \in(0, T) .
$$

Then, there exists at most one couple $\langle u, f\rangle \in \mathrm{L}^{\infty}\left((0, T), \mathrm{H}_{0}^{1}(\Omega)\right) \times \mathrm{L}^{2}(\Omega)$ with $\partial_{t}\left(g_{1} *\left(u-\tilde{u}_{0}\right)\right) \in$ $\mathrm{L}^{\infty}\left((0, T), \mathrm{L}^{2}(\Omega)\right)$, such that Problem (2), together with Condition (3), is satisfied. 
Proof. Let $h(t)>0$ with $h^{\prime}(t) \geqslant 0$ for all $t \in(0, T)$. We placed $\varphi=\tilde{H}(t) u(t)$ with $\tilde{H}(t):=\frac{1}{h(t)}$ in (7) and integrated over time $t \in(0, T)$ to obtain:

$$
\begin{aligned}
\int_{0}^{T} \tilde{H}(t)\left(\partial_{t}\left(g_{1} * u\right)(t), u(t)\right) \mathrm{d} t+\int_{0}^{T} \tilde{H}(t)\left\|A^{\frac{1}{2}}(t) \nabla u(t)\right\|^{2} \mathrm{~d} t & \\
& +\int_{0}^{T} \tilde{H}(t)\|\sqrt{c(t)} \nabla u(t)\|^{2} \mathrm{~d} t=0 .
\end{aligned}
$$

Employing integration in parts, we obtained:

$$
\begin{aligned}
\int_{0}^{T} \tilde{H}(t) & \left(\partial_{t}\left(g_{1} * u\right)(t), u(t)\right) \mathrm{d} t \\
& =\tilde{H}(T) \int_{0}^{T}\left(\partial_{t}\left(g_{1} * u\right)(t), u(t)\right) \mathrm{d} t-\int_{0}^{T} \tilde{H}^{\prime}(t)\left(\int_{0}^{t}\left(\partial_{t}\left(g_{1} * u\right)(s), u(s)\right) \mathrm{d} s\right) \mathrm{d} t .
\end{aligned}
$$

Note that $\tilde{H}^{\prime}(t)=-\frac{h^{\prime}(t)}{h(t)^{2}} \leqslant 0$ for all $t \in(0, T)$. From Lemma 2 for $n=1$, it followed that:

$$
0 \geqslant \int_{0}^{T} \tilde{H}(t)\left(\partial_{t}\left(g_{1} * u\right)(t), u(t)\right) \mathrm{d} t \geqslant \tilde{H}(T) \tilde{c}_{1} \int_{0}^{T}\|u(t)\|^{2} \mathrm{~d} t
$$

Therefore, $\int_{0}^{T}\|u(t)\|^{2} \mathrm{~d} t=0$ and, thus, $u=0$ a.e. in $Q_{T}$ and $f=0$ a.e. in $\Omega$.

If $h \in \mathrm{C}([0, T])$ satisfies $h(t) \geqslant h_{0}>0$, then the uniqueness of a solution to ISP (2) and (4) with $L(t)=L$ was established in [12] (Theorem 3.1). If $\partial_{t} u \in \mathrm{L}^{2}\left((0, T), \mathrm{H}_{0}^{1}(\Omega)\right)$, then $f(\mathbf{x})$ can be uniquely determined from (4) for general operator $L(t)$ assuming the monotonicity condition on $L(t)$ and $\left\{h>0, h^{\prime} \geqslant 0\right\}$ or $\left\{h<0, h^{\prime} \leqslant 0\right\}$ - see [13] (Theorem 3.1). Unfortunately, this assumption on $\partial_{t} u$ is typically not satisfied as mentioned before. The purpose of the next theorem was to show that [13] (Theorem 3.1) stayed valid if solution $u$ to Problem (2) satisfies $\partial_{t} u \in \mathrm{L}^{1}\left((0, T), \mathrm{H}_{0}^{1}(\Omega)\right)$ and $\partial_{t}\left(g_{1} *\left(u-\tilde{u}_{0}\right)\right)=g_{1} * \partial_{t} u \in \mathrm{L}^{\infty}\left((0, T), \mathrm{L}^{2}(\Omega)\right)$.

Theorem 3 (Uniqueness if $n=1$ using measurement (4)). Assume that $\psi_{T} \in \mathrm{L}^{2}(\Omega)$. Moreover, assume that $h \in \mathrm{C}^{1}((0, T])$ satisfies:

$$
h(t)>0, \quad h^{\prime}(t) \geqslant 0, \quad \forall t \in(0, T),
$$

or, either,

$$
h(t)<0, \quad h^{\prime}(t) \leqslant 0, \quad \forall t \in(0, T),
$$

and assume that:

$$
\xi^{T} \cdot\left(\partial_{t} A^{\frac{1}{2}}\right) A^{\frac{1}{2}} \xi \leqslant 0, \quad \forall \xi \in \mathbb{R}^{d} ; \quad \partial_{t} c(\cdot, t) \leqslant 0, \quad \forall t \in[0, T] .
$$

Then, there exists at most one couple $\langle u, f\rangle \in \mathrm{L}^{\infty}\left((0, T), \mathrm{H}_{0}^{1}(\Omega)\right) \times \mathrm{L}^{2}(\Omega)$ with $\partial_{t} u \in$ $\mathrm{L}^{1}\left((0, T), \mathrm{H}_{0}^{1}(\Omega)\right)$ and $g_{1} * \partial_{t} u \in \mathrm{L}^{\infty}\left((0, T), \mathrm{L}^{2}(\Omega)\right)$, such that Problem (2), together with Condition (4), is satisfied.

Proof. Since $\partial_{t} u(t) \in \mathrm{H}_{0}^{1}(\Omega)$ for a.a. $t \in(0, T)$, we could consider $\partial_{t} u$ as a test function, as conducted in [13] (Theorem 3.1). Lemma 1 stayed valid if $v \in \mathrm{L}^{1}\left((0, T), \mathrm{L}^{2}(\Omega)\right)$ and $g_{n} * v \in \mathrm{L}^{\infty}\left((0, T), \mathrm{L}^{2}(\Omega)\right)$. Hence, we could apply Lemma 1 for $n=1$ with $v=\partial_{t} u$, and all other steps stayed valid in the proof of [13] (Theorem 3.1) (note that all integrals stayed finite), i.e., we could conclude the current proof. 
Remark 1. The assumptions on solution $u$ in the previous theorem were fulfilled if the conditions (5) were satisfied. Indeed, we obtained:

$$
\int_{0}^{T}\left\|\partial_{t} u(t)\right\|_{\mathrm{H}_{0}^{1}(\Omega)} \mathrm{d} t \leqslant C T+C \int_{0}^{T} t^{\beta_{1}-1} \mathrm{~d} t \leqslant C
$$

and

$$
\begin{aligned}
& \max _{t \in[0, T]}\left\|\left(g_{1} * \partial_{t} u\right)(t)\right\| \leqslant C \max _{t \in[0, T]} \int_{0}^{t} \zeta(t-s) \mathrm{d} s+C \max _{t \in[0, T]} \int_{0}^{t} \zeta(t-s) s^{\beta_{1}-1} \mathrm{~d} s \leqslant C \\
& \text { as } \frac{t^{-\beta(\mathbf{x})}}{\Gamma(1-\beta(\mathbf{x}))} \leqslant \zeta(t):=\max \left\{1, t^{-\beta_{1}}\right\} \text { for } t>0 \text { and } \mathbf{x} \in \Omega .
\end{aligned}
$$

Remark 2. A counterexample (if h changed its sign) violating the uniqueness of a solution to the inverse problem considered in Theorem 3 could be found in [13] (Example 3.1). Whether the monotonicity conditions on $h$ can be relaxed in case of a non-autonomous operator $L$, as conducted in [7] for a classical diffusion equation, is still an open problem. Moreover, the recovery of $f(\mathbf{x})$ from (4) if $n=1$ without additional assumptions on the solution is also still an open problem.

\section{Inverse Source Problem for $n=2$}

In this section, we studied ISPs (2), (3) and (2), (4) for $n=2$. First, we mentioned the well-posedness of the forward problem [25] (Theorem 4.1).

Lemma 4 (Existence of a solution to the forward problem for $n=2$ ). Let the following assumptions be fulfilled:

- AS-1, AS-2 and AS-3;

- $\quad$ AS-6: $f \in \mathrm{L}^{\infty}\left((0, T), \mathrm{L}^{2}(\Omega)\right)$;

- AS-7: $\tilde{u}_{0} \in \mathrm{H}_{0}^{1}(\Omega)$ and $\tilde{v}_{0} \in \mathrm{L}^{2}(\Omega)$.

Then, there exists a unique weak solution $u$ to Problem (2) with $u \in C\left([0, T], \mathrm{L}^{2}(\Omega)\right) \cap$ $\mathrm{L}^{\infty}\left((0, T), \mathrm{H}_{0}^{1}(\Omega)\right), \partial_{t} u \in \mathrm{L}^{2}\left((0, T), \mathrm{L}^{2}(\Omega)\right)$ and $\partial_{t}\left(g_{2} *\left(\partial_{t} u-\tilde{v}_{0}\right)\right) \in \mathrm{L}^{\infty}\left((0, T), \mathrm{H}_{0}^{1}(\Omega)^{*}\right)$.

Now, both $u(\cdot, T)$ and $\int_{0}^{T} u(\cdot, t) \mathrm{d} t$ were well-defined as elements in $\mathrm{L}^{2}(\Omega)$. We first considered ISP (2) and (3) for $n=2$ with autonomous operator $L$.

Theorem 4 (Uniqueness if $n=2$ with $L(t)=L$ using measurement (3)). Let the conditions of Lemma 4 be satisfied. Assume that $U_{T} \in \mathrm{L}^{2}(\Omega)$. Moreover, assume that $h \in \mathrm{L}^{1}(0, T)$ satisfies:

$$
h(t)>0, \quad \forall t \in(0, T), \quad \text { or either } \quad h(t)<0, \quad \forall t \in(0, T) .
$$

Then, there exists at most one couple $\langle u, f\rangle \in\left[\mathrm{C}\left([0, T], \mathrm{L}^{2}(\Omega)\right) \cap \mathrm{L}^{\infty}\left((0, T), \mathrm{H}_{0}^{1}(\Omega)\right)\right] \times$ $\mathrm{L}^{2}(\Omega)$ with $\partial_{t} u \in \mathrm{L}^{2}\left((0, T), \mathrm{L}^{2}(\Omega)\right)$ and $\partial_{t}\left(g_{2} *\left(\partial_{t} u-\tilde{v}_{0}\right)\right) \in \mathrm{L}^{\infty}\left((0, T), \mathrm{H}_{0}^{1}(\Omega)^{*}\right)$, such that Problem (2), together with Condition (3), are satisfied.

Proof. We supposed that there were two solutions $\left\{u_{1}, f_{1}\right\}$ and $\left\{u_{2}, f_{2}\right\}$ satisfying (2) and (3). Then, the differences $u=u_{1}-u_{2}$ and $f=f_{1}-f_{2}$ satisfied the weak form:

$$
\left\langle\partial_{t}\left(g_{2} * \partial_{t} u\right)(t), \varphi\right\rangle+(A \nabla u(t), \nabla \varphi)+(c u(t), \varphi)=h(t)(f, \varphi),
$$


for all $\varphi \in \mathrm{H}_{0}^{1}(\Omega)$ with $u(\cdot, 0)=0, \partial_{t} u(\cdot, 0)=0$ and $\int_{0}^{T} u(\cdot, s) \mathrm{d} s=0$ in $\Omega$. Next, we integrated in time over $t \in(0, \eta) \subset(0, T)$. Again, we placed $\Phi_{h}(t):=\int_{0}^{t} h(s) \mathrm{d} s$. We obtained:

$$
\left(\left(g_{2} * \partial_{t} u\right)(\eta), \varphi\right)+\left(A \int_{0}^{\eta} \nabla u(t) \mathrm{d} t, \nabla \varphi\right)+\left(c \int_{0}^{\eta} u(t) \mathrm{d} t, \varphi\right)=\Phi_{h}(\eta)(f, \varphi) .
$$

We only considered the case $h(t)>0$ for all $t \in(0, T)$ and defined $H(\eta):=\frac{1}{\Phi_{h}(\eta)}$. We took $\varphi=H(\eta) u(\eta)$ in (13) and integrated in time over $\eta \in(0, T)$ to obtain:

$$
\begin{aligned}
\int_{0}^{T} H(\eta)\left(\partial_{t}\left(g_{2} * u\right)(\eta), u(\eta)\right) \mathrm{d} \eta+\int_{0}^{T} H(\eta)( & \left.A \int_{0}^{\eta} \nabla u(t) \mathrm{d} t, \nabla u(\eta)\right) \mathrm{d} \eta \\
& +\int_{0}^{T} H(\eta)\left(c \int_{0}^{\eta} u(t) \mathrm{d} t, u(\eta)\right) \mathrm{d} \eta=0 .
\end{aligned}
$$

We only pointed out how to handle the first term on the LHS. The other terms can be handled as in Theorem 1. Using integration by parts, we obtained:

$$
\begin{aligned}
& \int_{0}^{T} H(\eta)\left(\partial_{t}\left(g_{2} * u\right)(\eta), u(\eta)\right) \mathrm{d} \eta \\
& \quad=H(T) \int_{0}^{T}\left(\partial_{t}\left(g_{2} * u\right)(\eta), u(\eta)\right) \mathrm{d} \eta-\int_{0}^{T} H^{\prime}(\eta)\left(\int_{0}^{\eta}\left(\partial_{t}\left(g_{2} * u\right)(t), u(t)\right) \mathrm{d} t\right) \mathrm{d} \eta .
\end{aligned}
$$

Therefore, by Lemma 2 for $n=2$, we obtained:

$$
0 \geqslant \int_{0}^{T} H(\eta)\left(\partial_{t}\left(g_{2} * u\right)(\eta), u(\eta)\right) \mathrm{d} \eta \geqslant H(T) \tilde{c}_{2} \int_{0}^{T}\|u(t)\|^{2} \mathrm{~d} t,
$$

from which we concluded the proof.

The uniqueness of a solution to ISP (2) and (3) for a time-dependent operator $L$ is still an open problem. When we assumed a higher regularity on the solution of Problem (2) (in comparison with Lemma 4), we were able to show the following theorem concerning ISP (2) and (4). Hence, the recovery of $f(\mathbf{x})$ from Measurement (4) if $n=2$ without additional assumptions on the solution is still an open problem.

Theorem 5 (Uniqueness if $n=2$ using measurement (4)). Assume that $\psi_{T} \in \mathrm{L}^{2}(\Omega)$ and:

$$
\xi^{T} \cdot\left(\partial_{t} A^{\frac{1}{2}}\right) A^{\frac{1}{2}} \xi \leqslant 0, \quad \forall \xi \in \mathbb{R}^{d} ; \quad \partial_{t} c(\cdot, t) \leqslant 0, \quad \forall t \in(0, T) .
$$

Moreover, assume that $h \in \mathrm{C}^{1}((0, T])$ satisfies:

$$
h(t)>0, \quad h^{\prime}(t) \geqslant 0, \quad \forall t \in(0, T),
$$

or, either,

$$
h(t)<0, \quad h^{\prime}(t) \leqslant 0, \quad \forall t \in(0, T) .
$$

Then, there exists at most one couple $\langle u, f\rangle \in\left[\mathrm{C}\left([0, T], \mathrm{L}^{2}(\Omega)\right) \cap \mathrm{L}^{\infty}\left((0, T), \mathrm{H}_{0}^{1}(\Omega)\right)\right] \times$ $\mathrm{L}^{2}(\Omega)$ with $\partial_{t} u \in \mathrm{L}^{2}\left((0, T), \mathrm{H}_{0}^{1}(\Omega)\right)$ and $\partial_{t}\left(g_{2} *\left(\partial_{t} u-\tilde{v}_{0}\right)\right) \in \mathrm{L}^{\infty}\left((0, T), \mathrm{L}^{2}(\Omega)\right)$, such that Problem (2), together with Condition (4), are fulfilled.

Proof. We supposed that there were two solutions $\left\{u_{1}, f_{1}\right\}$ and $\left\{u_{2}, f_{2}\right\}$ satisfying (2) and (4). Then, the differences $u=u_{1}-u_{2}$ and $f=f_{1}-f_{2}$ satisfied (12) for all $\varphi \in \mathrm{H}_{0}^{1}(\Omega)$ with $u(\cdot, 0)=0, \partial_{t} u(\cdot, 0)=0$ and $u(\cdot, T)=0$ in $\Omega$. Let $h(t)>0$ with $h^{\prime}(t) \geqslant 0$ for all $t \in(0, T)$. 
Define $\tilde{H}(t):=\frac{1}{h(t)}$. Here, we chose $\varphi=\tilde{H}(t) \partial_{t} u(t)$ in (13) and integrated in time over $t \in(0, T)$ to obtain:

$$
\begin{aligned}
\int_{0}^{T} \tilde{H}(t)\left(\partial_{t}\left(g_{2} * \partial_{t} u\right)(t), \partial_{t} u(t)\right) \mathrm{d} t+\int_{0}^{T} \tilde{H}(t)\left(A \nabla u(t), \nabla \partial_{t} u(t)\right) \mathrm{d} t \\
\quad+\int_{0}^{T} \tilde{H}(t)\left(c u(t), \partial_{t} u(t)\right) \mathrm{d} t=\int_{0}^{T}\left(f, \partial_{t} u(t)\right) \mathrm{d} t=(f, u(T)-u(0))=0
\end{aligned}
$$

The second and third terms on the LHS were positive - see the proof of [13] (Theorem 3.1). For the first term, we, again, employed integration by parts and Lemma 2 for $n=2$ to obtain:

$$
0 \geqslant \int_{0}^{T} \tilde{H}(t)\left(\partial_{t}\left(g_{2} * \partial_{t} u\right)(t), \partial_{t} u(t)\right) \mathrm{d} t \geqslant \tilde{H}(T) \tilde{c}_{2} \int_{0}^{T}\left\|\partial_{t} u(t)\right\|^{2} \mathrm{~d} t .
$$

It followed that $u=0$ in $Q_{T}$ as $\partial_{t} u(\cdot, 0)=0$ in $\Omega$. We concluded the proof as before.

Remark 3. The assumptions on solution $u$ in the previous theorem were fulfilled if the conditions (6) were satisfied.

Remark 4. Important to note is that no additional damping term in the fractional wave equation was needed in order to obtain the uniqueness to ISP (2) and (4) for $n=2$, which was the case for the classical wave equations [6].

\section{Conclusions}

The purpose of the current study was to determine which type of measurement was suited to recover, in a unique way, a space-dependent source $f(\mathbf{x})$, taking into account the typical behaviour of the solution to FPDEs. The coefficients in the governing operator $L$ might be time-dependent. Case $n=1$ corresponded with a fractional diffusion equation of the space-dependent variable order, whilst case $n=2$ corresponded with the fractional wave analogue.

For a non-autonomous operator $L(t)$, and under low regularity assumptions (see Lemmas 3 and 4), the time-averaged Measurement (3) was well-defined as an element in $\mathrm{L}^{2}(\Omega)$ for $n=1$, whilst both Measurements (3) and (4) were well-defined for $n=2$. This observation suggested that the time-averaged Measurement (3) was better suited to recover the space-dependent source $f(\mathbf{x})$ if $n=1$.

In Theorem 1, we showed that $f(\mathbf{x})$ could be uniquely determined from Measurement (3) if $L(t)=L$ assuming that $h \in \mathrm{L}^{1}(0, T)$ satisfied either $h>0$ or $h<0$. In the case of a non-autonomous operator $L(t)$, we needed additional assumptions on the solution and monotonicity conditions on $h \in \mathrm{C}^{1}((0,1])$ (i.e., $\left\{h>0, h^{\prime} \geqslant 0\right\}$ or $\left.\left\{h<0, h^{\prime} \leqslant 0\right\}\right)$ to obtain the uniqueness of a solution to ISP (2) and (3). No extra conditions on $L(t)$ were needed-see Theorem 2 for more details. In Theorem 3, we extended the result from [13] (Theorem 3.1) to solutions $u$ of Problem (2), satisfying Bound (5). ISP (2) and (4) had a unique solution under appropriate conditions on $u$, monotonicity condition on $L(t)$ and $\left\{h>0, h^{\prime} \geqslant 0\right\}$ or $\left\{h<0, h^{\prime} \leqslant 0\right\}$. Concerning Theorems 2 and 3 , whether the assumptions on $u$ and/or the monotonicity condition on $h$ can be further relaxed, is still an open problem. This gives a first direction for future research.

The uniqueness of a solution to ISP (2) and (3) for $n=2$ was only obtained for an autonomous operator $L$ if $h \in \mathrm{L}^{1}(0, T)$ satisfied either $h>0$ or $h<0$-see Theorem 4. Hence, the same problem for a time-dependent operator $L(t)$ is still unsolved. Finally, Theorem 5 concerned the uniqueness of a solution to ISP (2) and (4) for $n=2$. The monotonicity conditions on $L(t)$ and $h$ were the same as in Theorem 3. Furthermore, here, the question raised by this study was whether it was possible to relax the conditions on the solution and/or $h$. Important to note is that, for this latter ISP, no additional damping term in the governing equation was needed, as was the case for the wave equations with classical derivatives. Moreover, we also addressed that, for the fractional wave equation, the typical 
behaviour of Solution (6) was satisfied, and that it might be possible that the final in time Measurement (4) was more suited to recover the space-dependent source $f(\mathbf{x})(n=2)$.

Finally, we mentioned that the obtained results were also valid if the order of the fractional derivative was constant.

Funding: The author was supported by a postdoctoral fellowship of the Research FoundationFlanders (106016/12P2919N).

Institutional Review Board Statement: Not applicable.

Informed Consent Statement: Not applicable.

Conflicts of Interest: The authors declare no conflict of interest.

\section{References}

1. Almeida, R.; Tavares, D.; Torres, D.F.M. The Variable-Order Fractional Calculus of Variations; Springer: Cham, Switzerland, 2019; p. $x i v+124$.

2. Van Bockstal, K. Existence of a unique weak solution to a non-autonomous time-fractional diffusion equation with space-dependent variable order. Adv. Differ. Equ. 2021, 2021, 314. [CrossRef]

3. Johansson, B.T.; Lesnic, D. A variational method for identifying a spacewise-dependent heat source. IMA J. Appl. Math. 2007, 72, 748-760. [CrossRef]

4. Erdem, A.; Lesnic, D.; Hasanov, A. Identification of a spacewise dependent heat source. Appl. Math. Model. 2013, 37, 10231-10244. [CrossRef]

5. D'haeyer, S.; Johansson, B.T.; Slodička, M. Reconstruction of a spacewise-dependent heat source in a time-dependent heat diffusion process. IMA J. Appl. Math. 2014, 79, 33-53. [CrossRef]

6. Van Bockstal, K.; Slodička, M. Recovery of a space-dependent vector source in thermoelastic systems. Inverse Probl. Sci. Eng. 2015, 23, 956-968. [CrossRef]

7. Slodička, M.; Johansson, B.T. Uniqueness and counterexamples in some inverse source problems. Appl. Math. Lett. 2016, 58, 56-61. [CrossRef]

8. Lesnic, D.; Hussein, S.O.; Johansson, B.T. Inverse space-dependent force problems for the wave equation. J. Comput. Appl. Math. 2016, 306, 10-39. [CrossRef]

9. Van Bockstal, K. Identification of an unknown spatial load distribution in a vibrating beam or plate from the final state. J. Inverse -Ill-Posed Probl. 2019, 27, 623-642. [CrossRef]

10. Slodička, M. Uniqueness for an inverse source problem of determining a space dependent source in a non-autonomous parabolic equation. Appl. Math. Lett. 2020, 107, 106395. [CrossRef]

11. Sakamoto, K.; Yamamoto, M. Inverse source problem with a final overdetermination for a fractional diffusion equation. Math. Control Relat. Fields 2011, 1, 509-518. [CrossRef]

12. Wei, T.; Wang, J. A modified quasi-boundary value method for an inverse source problem of the time-fractional diffusion equation. Appl. Numer. Math. 2014, 78, 95-111. [CrossRef]

13. Slodička, M.; Šišková, K.; Van Bockstal, K. Uniqueness for an inverse source problem of determining a space dependent source in a time-fractional diffusion equation. Appl. Math. Lett. 2019, 91, 15-21. [CrossRef]

14. Yan, X.B.; Wei, T. Determine a Space-Dependent Source Term in a Time Fractional Diffusion-Wave Equation. Acta Appl. Math. 2020, 165, 163-181. [CrossRef]

15. Jiang, S.Z.; Wu, Y.J. Recovering space-dependent source for a time-space fractional diffusion wave equation by fractional Landweber method. Inverse Probl. Sci. Eng. 2021, 29, 990-1011. [CrossRef]

16. Slodička, M. Uniqueness for an inverse source problem of determining a space-dependent source in a non-autonomous time-fractional diffusion equation. Fract. Calc. Appl. Anal. 2020, 23, 1702-1711. [CrossRef]

17. Stynes, M. Too much regularity may force too much uniqueness. Fract. Calc. Appl. Anal. 2016, 19, 1554-1562. [CrossRef]

18. Stynes, M.; O’Riordan, E.; Gracia, J.L. Error Analysis of a Finite Difference Method on Graded Meshes for a Time-Fractional Diffusion Equation. SIAM J. Numer. Anal. 2017, 55, 1057-1079. [CrossRef]

19. Otárola, E.; Salgado, A.J. Regularity of solutions to space-time fractional wave equations: A PDE approach. Fract. Calc. Appl. Anal. 2019, 21, 1262-1293. [CrossRef]

20. Kopteva, N. Error analysis of the $L 1$ method on graded and uniform meshes for a fractional-derivative problem in two and three dimensions. Math. Comput. 2019, 88, 2135-2155. [CrossRef]

21. Roubíček, T. Nonlinear Partial Differential Equations with Applications; ISNM, Birkhäuser Verlag: Basel, Switzerland, 2005; Volume 153.

22. Koeber, M.; Schäfer, U. The unique square root of a positive semidefinite matrix. Int. J. Math. Educ. Sci. Technol. 2006, 37, 990-992. [CrossRef]

23. Gripenberg, G.; Londen, S.; Staffans, O. Volterra Integral and Functional Equations; Cambridge Ocean Technology Series; Cambridge University Press: Cambridge, UK, 1990. 
24. Zeidler, E. Nonlinear Functional Analysis and Its Applications II/A: Linear Monotone Operators; Springer: Berlin/Heidelberg, Germany, 1990.

25. Van Bockstal, K.; Hendy, A.S.; Zaky, M.A. Space-dependent variable-order time-fractional wave equation: Existence and uniqueness of its weak solution via time discretization method. Adv. Differ. Equ. 2021, 2021, 1-43. 